\title{
The role of osmotic self-inflatable tissue expanders in intestinal transplant candidates 2 , 论访
}

\author{
Laurens J. Ceulemans ${ }^{\mathrm{a}, *}$, Nathalie P. Deferm ${ }^{\mathrm{a}}$, Marc Miserez ${ }^{\mathrm{b}}$, Francesca Maione ${ }^{\mathrm{a}}$, \\ Diethard Monbaliu ${ }^{a}$, Jacques Pirenne ${ }^{a}$
}

a Abdominal Transplant Surgery, University Hospitals Leuven, \&' Department of Microbiology and Immunology, KU Leuven, Belgium

b Abdominal Surgery, University Hospitals Leuven, \& Department of Development and Regeneration, KU Leuven, Belgium

\section{A B S T R A C T}

Intestinal transplantation (ITx) is often associated with decreased abdominal domain, rendering abdominal closure difficult. Pre-transplant placement of tissue expanders (TE) can overcome this challenge; however it can be associated with life-threatening complications. This review aimed to comprehensively summarize all available literature on TE in ITx candidates and include the technical details of osmotic, self-inflatable TE -a technique undescribed before. PubMed, EMBASE and CCTR were searched until April 30, 2016. Based on structured data abstraction and detailed analysis, eighteen cases of TE (inflatable) in ITx candidates were found. Localisation of placement was: subcutaneously in 11; intraperitoneally in $4 ; 1$ patient had 1 TE placed retromuscularly and 1 intraperitoneally; 1 patient had biplanar TE (intraperitoneally placed and extending retromuscularly) and in 1 localisation was unreported. Complication rate was high (61\%), injection- or intraperitoneal-related, resulting in life-threatening infections/hematoma. With successful expansion, physiological graft protection -by skin $+/$-fascia- was always achieved. In completion of this review, we describe our own experience with two patients (7.5-, 34-year-old females), in whom osmotic TE were placed subcutaneously pre-ITx. No TE-related complications occurred and both patients underwent uncomplicated ITx with respectively primary skin and skin + fascia closure. The pros and cons of each TE type and placement are discussed, resulting in the overall conclusions that TE offer an important benefit in graft-protection following ITx. Osmotic TE are safer than conventional prostheses by avoiding percutaneous injections. Subcutaneous placement seems to be safer and more reliable.

(c) 2016 Elsevier Inc. All rights reserved.

\section{Introduction}

Intestinal transplantation (ITx) is the least common form of organ transplantation and often deemed one of the most difficult. It is only offered to selected patients with chronic intestinal failure -like short bowel syndrome (SBS)- and life-threatening complications of nutritional support such as impaired venous access, recurrent infections and liver failure [1,2]. Patients with a SBS after (repetitive) surgical resection of the entire small bowel often present with an impaired abdominal skin and fascia elasticity and markedly decreased intra-abdominal domain, which is even more intensified at the moment of ITx by reperfusion

\footnotetext{
Authorship: LJC, NPD: designed the paper, reviewed the literature, collected and analyzed the data, contributed important ideas, wrote the paperFM, DM: reviewed the literature, contributed important ideas, revised the paperMM, JP: designed the paper, contributed important ideas, expert in the clinical field, revised the paper.

is

* Corresponding author at: Abdominal Transplant Surgery, University Hospitals Leuven, Herestraat 49, B-3000 Leuven, Belgium. Tel.: +32 16348727 ; fax: +32 16348743.

E-mail address: ceulemans.laurens@gmail.com (L.J. Ceulemans).
}

edema of the graft and the recipient. All these factors render abdominal closure after ITx extremely challenging, in particular in children [3,4]. However, successful closure in this mostly very ill and highly immunosuppressed population has proven to be essential to decrease the risk of infections, fistulisations and mycotic aneurysms and to improve graft and patient survival $[5,6]$. Although the preferred donor to recipient weight ratio (DRWR) should be lower than 1 to increase the possibility of primary abdominal closure, very few small size donors are available [7]. This critical shortage of size-matched organs, particularly for infants in need of combined liver-intestinal transplantation (CLi-ITx), has led to long waiting times and pre-transplant mortality rates up to 50\% [8]. In order to transplant larger grafts and expand the donor pool, several techniques have been developed, like surgical reduction of the graft, intra-operative bridging of the abdominal fascia (with synthetic/ biological meshes or cadaveric donor grafts), component separation techniques, rotation/free flap coverage and even abdominal wall transplantation [4,9-12].

Pre-transplant placement of inflatable tissue expanders (TE) has only been reported in a few cases [13-18]. They offer the potential benefit to increase the abdominal domain pre-ITx and provide sufficient skin and/or fascia resulting in physiological graft protection without the aid of foreign material. However, serial percutaneous fluid injections 
expose the patients to the risk of serious complications like infection or hematoma. This review aimed (i) to comprehensively summarize all available literature on TE in ITx candidates and (ii) to report our own experience with two cases in which osmotic self-inflatable TE were used -a technique undescribed before- which has the major benefit of avoiding serial percutaneous injections and its associated complications.

\section{Materials and methods}

\subsection{Study selection}

To identify papers relevant to the role of TE in ITx, a literature search of three major electronic databases - MEDLINE (via PubMed), EMBASE and Cochrane Controlled Trial Reports - was conducted until April 30th, 2016, based on three concepts: 1) Transplantation; 2) Intestine; and 3) TE or abdominal wound closure techniques. Study inclusion criteria were: original papers reporting TE in ITx candidates; quantitative or qualitative studies; literature written in English, Dutch, French or German. Exclusion criteria were: abstracts, editorials and opinions. Two reviewers (LJC and NPD) screened all titles and abstracts independently for eligibility and cross-checked the references of the included papers.

\subsection{Data extraction}

Data extraction included information on the author, year of publication, patient age, time of TE placement, type of ITx, donor-recipient weight ratio (DRWR), number of TE, total volume of TE, localisation (subcutaneously, retromuscular or intraperitoneally), type of TE (inflatable or osmotic), TE-related complications, number of removed TE and successful primary abdominal closure after ITx. Data were extracted independently by two researchers (LJC and NPD) and tabulated descriptively using Microsoft Excel® (Microsoft Office 2013).

Based on a detailed analysis of all reported cases, we discuss the pros and cons of each TE type and placement.

\subsection{New technique of osmotic tissue expanders}

In addition to the reported cases in literature, we added our experience, in which we used osmotic TE (Osmed ${ }^{\mathrm{Gmbh}}$, Ilmenau, Germany) that were inserted subcutaneously via small incisions - a technique undescribed before. Although this placement would only result in skin expansion without enlarging the abdominal fascia, it was considered the safest technique that would offer immediate graft protection postITx and that would avoid serial percutaneous injections and its associated complications. Informed consent of the patient or the responsible parent was obtained. Once implanted, the dehydrated hydrogel expanded by absorbing body fluid in the first $6-8$ weeks, leading to gradual expansion of the skin without the need for external fluid injection. To avoid overexpansion, TE were self-limiting in size.

\section{Results}

\subsection{Descriptive analysis}

Six papers were finally included of which data abstraction is reported in detail in Table 1[13-18].

The use of TE prior to ITx was first reported by de Ville de Goyet et al. in 2000 [13]. In an 18 month-old child ( $9.8 \mathrm{~kg}$ ) with short gut and tight abdomen, insertion of a preperitoneally placed (no other localisation details were provided) inflatable TE allowed sufficient abdominal expansion. Waiting time after implantation was 3 months and a surgically reduced cLi-ITx graft from a larger donor ( 12 years of age; $35 \mathrm{~kg}$; DRWR: 3.6) could be transplanted with primary skin and fascia closure.

In the same year, Alexandrides et al. reported on a 19 year-old male in whom 2 inflatable TE were inserted intraperitoneally [14]. However, the patient developed TE-related peritonitis and the expanders had to be removed 18 days later.

In 2008, Marin-Gutzke et al. described the combined placement of an intraperitoneal $\left(1000 \mathrm{~cm}^{3}\right)$ and retromuscular $\left(700 \mathrm{~cm}^{3}\right)$ prosthesis with subcutaneous ports for percutaneous inflation in a 27 -year-old patient awaiting isolated ITx [15]. Increase of the abdominal cavity was required after total small bowel resection for an abdominal desmoid tumor. One month after insertion, expansion was completed and 4 months later ITx could be performed uneventfully. The patient tolerated the TE without complications and the abdominal cavity created was large enough to accommodate the bowel. Tension-free fascia closure could be achieved.

In 2013, Watson et al. reported on the Cleveland experience in which 9 inflatable TE were inserted subcutaneously in 4 adult patients [16]. Although the second patient lost 1 of his 3 expanders due to port-related dysfunction, primary abdominal closure could be obtained (no details on skin or fascia closure were provided). In 2 other patients primary abdominal closure could be achieved, reported as skin closure in combination with an Alloderm mesh to bridge the fascia in the first and both skin and fascia closure in the second patient. The fourth patient of this cohort was still on the waiting list at the time of report.

Vidyadharan et al. described in 2013 the Birmingham experience in which 7 children awaiting cLi-ITx received 17 subcutaneously placed inflatable TE [17]. Although the volume of each expander was reported, this could not be linked to the patient, resulting in an overall mean volume of $278 \mathrm{~cm}^{3} /$ TE. All patients suffered from TE-related complications and 1 patient died from a TE injection-related infection. Two other patients died before transplantation (TE-unrelated). All 4 survivors underwent a reduced cLi-ITX, and required staged abdominal closure. No further details were provided. In the discussion of the paper the authors reported their experience with intraperitoneal placement of TE in 3 additional patients (the number of TE was not described). Two of them developed hematoma and 1 compression of the inferior caval vein.

Weiner et al. introduced in 2014 in a 34 month-old child - with SBS due to volvulus - the use of 2 biplanar TE, intraperitoneally placed and extending retromuscularly into the chest wall and groin, respectively [18]. Serial injections over 3 months, resulted in a tolerated expansion of 230 and $345 \mathrm{~cm}^{3}$. Ten months after placement, the patient underwent a successful isolated ITx. Although DRWR was limited to 0.9 , post-reperfusion edema of the graft required temporarily closure of the abdominal wall with a Gore-Tex mesh for 7 days, after which fascia closure could be obtained. The extension of the biplanar TE into retromuscular pockets allowed the TE to be supported and anchored by stable skeletal structures (ribs and iliac crest) minimizing the risk of migration and redirecting the force of expansion outward. The latter, hypothetically, avoided pressure on the peritoneal content.

\subsection{Osmotic tissue expanders}

The first patient in our own experience was a 7,5 year-old girl, who suffered from a neonatal volvulus, requiring repeated surgical resections of the small bowel and ascending colon leaving her with an ultrashort bowel and duodenostomy. She was completely dependent on total parenteral nutrition and gradually developed liver cirrhosis with portal hypertension and hemorrhagic gastro- and duodenopathy. The only remaining option was cLi-ITx. In order to have the possibility to accept larger grafts and offer immediate physiological graft protection post-ITx, three TE were inserted subcutaneously: the first $\left(450 \mathrm{~cm}^{3}\right)$ in the right hypochondrium; the second $\left(300 \mathrm{~cm}^{3}\right)$ in the left hypochondrium - adjacent to the stoma; and the third $\left(600 \mathrm{~cm}^{3}\right)$ in the right fossa (Fig. 1A1). Although the operation was uneventful, the left TE had to be removed two days later due to stoma-bag application difficulties. The remaining TE reached their maximum volume and no other complications occurred. 18 months later (weight: $25 \mathrm{~kg}$ ) the patient underwent cLi-ITx from a 9-year-old male deceased donor 
Table 1

Literature review on tissue expanders in intestinal transplantation.

\begin{tabular}{|c|c|c|c|c|c|c|c|c|c|c|c|c|}
\hline Author & Year & Patient age & $\begin{array}{l}\text { Time } \\
\text { pre- Tx }\end{array}$ & Type Tx & DRWR $^{\dagger}$ & $\mathrm{TE}^{+}$ & Total volume (cc) & Localization TE & $\begin{array}{l}\text { Type TE: } \\
\text { Inflatable/Osmotic }\end{array}$ & $\begin{array}{l}\text { TE } \\
\text { compli-cations }\end{array}$ & TE removed & $\begin{array}{l}\text { Primary abdominal closure: } \\
\text { Yes/No, Skin/Fascia }\end{array}$ \\
\hline $\begin{array}{l}\text { de Ville de } \\
\text { Goyet }\end{array}$ & 2000 & $1 \mathrm{y} 6 \mathrm{mo}$ & $3 \mathrm{mo}$ & c Li-ITx* (reduced graft) & 3,6 & 1 & 220 & Extraperitoneal & Inflatable & None & 0 & Yes, Skin + Fascia \\
\hline Alexandrides & 2000 & $19 \mathrm{y}$ & $1 \mathrm{y} 5 \mathrm{mo}$ & $\operatorname{MvTx}^{\dagger}$ & 1,8 & 2 & $450 / 250$ & Intraperitoneal & Inflatable & $\operatorname{Inf}^{\S}$ & 2 & Yes, NA (TE removed) \\
\hline Marin-Gutzke & 2002 & $27 y$ & $5 \mathrm{mo}$ & $\mathrm{ITx}^{\ddagger}$ & $N A$ & 2 & $1000 / 700$ & $\begin{array}{l}\text { Intraperitoneal } \\
\text { (1) Retromuscular (1) }\end{array}$ & Inflatable & None & 0 & Yes, Skin + Fascia \\
\hline \multirow[t]{4}{*}{ Watson } & \multirow[t]{4}{*}{2013} & $32 \mathrm{y}$ & $1 \mathrm{y} 6 \mathrm{mo}$ & ITx & NA & 2 & $480 / 480$ & Subcutaneous & Inflatable & None & 0 & Yes, Skin (Fascia: Alloderm) \\
\hline & & $47 \mathrm{y}$ & $5 \mathrm{mo}$ & ITx & NA & 3 & $N A(1) / 532 / 118$ & Subcutaneous & Inflatable & Port-related & 1 & Yes, $N A$ \\
\hline & & $44 y$ & $2 \mathrm{y} 3 \mathrm{mo}$ & Re-ITx & NA & 2 & $753 / 608$ & Subcutaneous & Inflatable & None & 0 & Yes, Skin + Fascia \\
\hline & & $56 y$ & i & $W L^{\#}$ & 1 & 2 & $545 / 746$ & Subcutaneous & Inflatable & None & 0 & 1 \\
\hline \multirow[t]{7}{*}{ Vidyadharan } & \multirow[t]{7}{*}{2013} & $1 \mathrm{y} 3 \mathrm{mo}$ & $5 \mathrm{mo}$ & c Li-ITx (reduced graft) & 2,6 & 3 & mean:278/TE ${ }^{\circ}$ & Subcutaneous & Inflatable & Hem & 0 & NA \\
\hline & & $10 \mathrm{mo}$ & $4 \mathrm{mo}$ & c Li-ITx (reduced graft) & 1,7 & 3 & mean:278/TE & Subcutaneous & Inflatable & Port-related & 0 & NA \\
\hline & & $11 \mathrm{mo}$ & $6 \mathrm{mo}$ & c Li-ITx (reduced graft) & 1,5 & 2 & mean:278/TE & Subcutaneous & Inflatable & Hem/dehis ${ }^{\wedge}$ & 1 & NA \\
\hline & & $11 \mathrm{mo}$ & $1 \mathrm{y} 1 \mathrm{mo}$ & c Li-ITx (reduced graft) & 1,3 & 1 & mean:278/TE & Subcutaneous & Inflatable & Hem/dehis & 0 & NA \\
\hline & & $1 \mathrm{y} 2 \mathrm{mo}$ & i & Died on WL & 1 & 3 & mean:278/TE & Subcutaneous & Inflatable & Hem/dehis & 1 & 1 \\
\hline & & $1 \mathrm{y} 2 \mathrm{mo}$ & l & Died on WL & i & 3 & mean:278/TE & Subcutaneous & Inflatable & Hem/inf/death & 1 & l \\
\hline & & $1 \mathrm{y} 3 \mathrm{mo}$ & / & Died on WL & 1 & 2 & mean:278/TE & Subcutaneous & Inflatable & Hem & 0 & / \\
\hline (discussion) & & $N A^{q}$ & NA & NA & NA & $\geq 1$ & NA & Intraperitoneal & Inflatable & Hem & NA & NA \\
\hline (discussion) & & NA & NA & NA & NA & $\geq 1$ & NA & Intraperitoneal & Inflatable & Hem & NA & NA \\
\hline (discussion) & & $N A$ & $N A$ & $N A$ & $N A$ & $\geq 1$ & $N A$ & Intraperitoneal & Inflatable & $\begin{array}{l}\text { ICV }^{£} \\
\text { compression }\end{array}$ & $N A$ & NA \\
\hline Weiner & 2014 & $2 \mathrm{y} 10 \mathrm{mo}$ & $10 \mathrm{mo}$ & ITx & 0.9 & 2 & $230 / 345$ & $\begin{array}{l}\text { Biplanar: } \\
\text { Intraperitoneal + Retromuscular }\end{array}$ & Inflatable & None & 0 & Yes, Skin (Fascia: Gore-Tex) \\
\hline \multirow[t]{2}{*}{ Ceulemans } & \multirow[t]{2}{*}{2015} & $7 \mathrm{y} 6 \mathrm{mo}$ & $1 \mathrm{y} 5 \mathrm{mo}$ & c Li-ITx & 1,2 & 3 & $450 / 300 / 600$ & Subcutaneous & Osmotic & Stoma-related & 1 & Yes, Skin \\
\hline & & $34 \mathrm{y}$ & $2 \mathrm{mo}$ & ITx (Living) & 1,2 & 2 & $400 / 400$ & Subcutaneous & Osmotic & None & 0 & Yes, Skin + Fascia \\
\hline
\end{tabular}

\begin{tabular}{clllll}
\hline $\begin{array}{c}\text { Median } \\
\text { (range) }\end{array}$ & $2 \mathrm{y} 10 \mathrm{mo}$ & $6 \mathrm{mo}$ & 1.5 & 2 & $278 \mathrm{~cm}^{3} / \mathrm{TE}$ \\
& $(10 \mathrm{mo}-56 \mathrm{y})$ & $(2 \mathrm{mo}-2 \mathrm{y}$ & $(0.9-3.6)$ & $(1-3)$ & $\left(118-1000 \mathrm{~cm}^{3}\right)$
\end{tabular}

Total Patients: 20 Grafts: c Li-ITx: 6 ITx:

Grafts: c Li-ITx: 6 ITx:
6MvTx: 1 Died/WL: 4 $(0.9-3.6) \quad(1-3) \quad\left(118-1000 \mathrm{~cm}^{3}\right)$

Tota

$T E: \geq$
41

,

Patients: Subcutaneous: 13

traperitoneal: 4

Retromuscular \&

Biplanar: 1

Extraperitoneal:1

\section{Patients:}

Inflatable: 18
Osmotic: 2

.



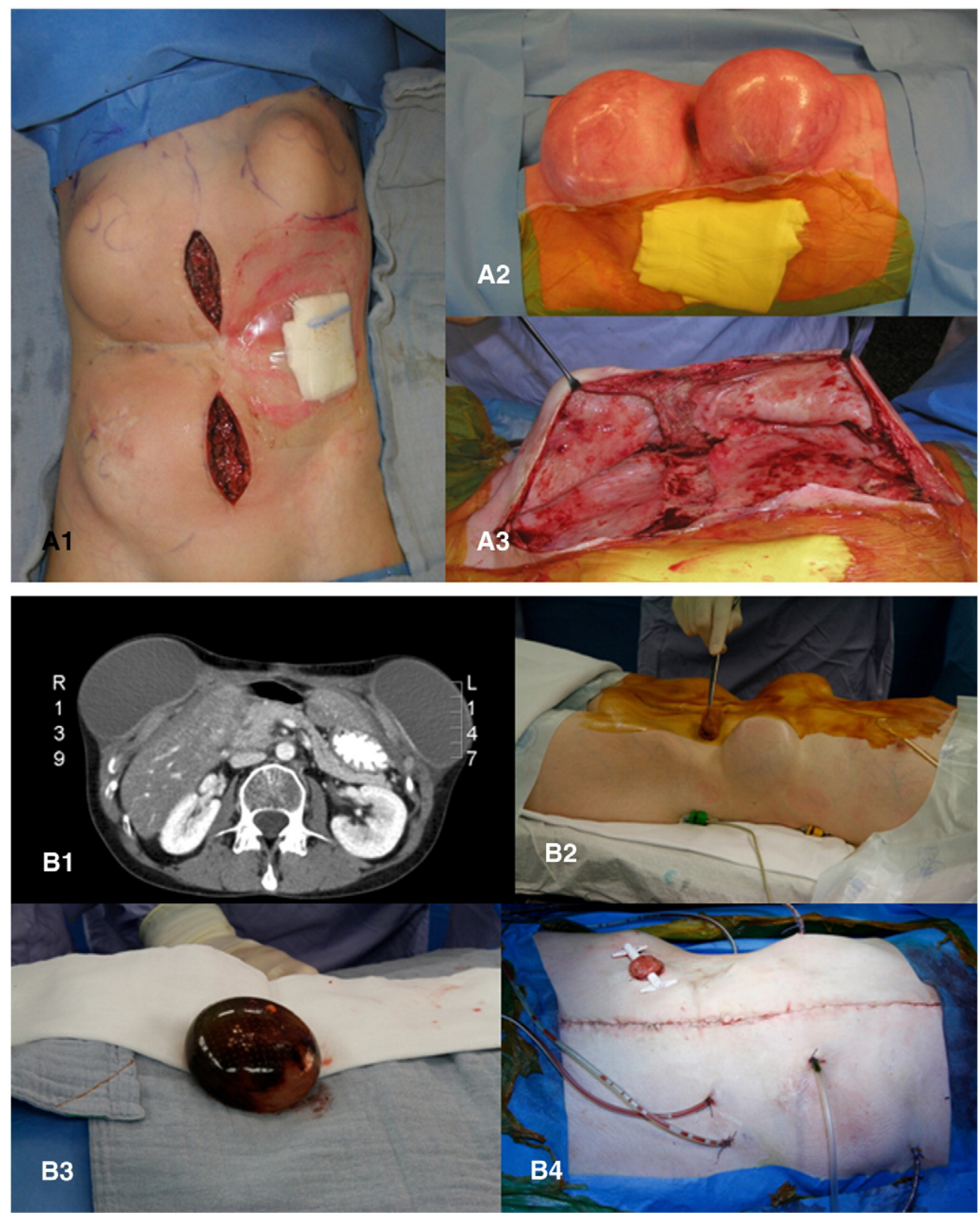

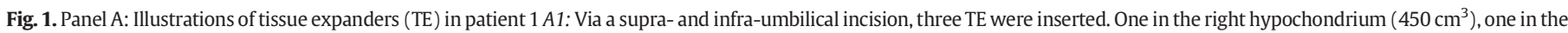

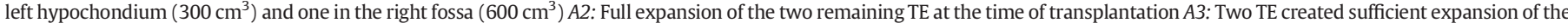

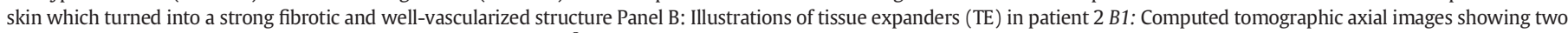

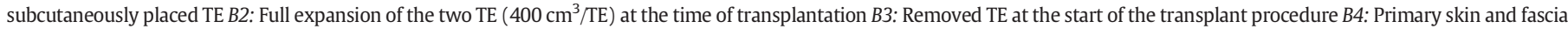
closure of the abdominal wall was achieved after transplantation.

(weight: $30 \mathrm{~kg}$ ) (DRWR: 1.2). Both TE were removed at the start of the procedure. The prostheses had turned the skin into a strong fibrotic layer that easily enabled primary skin closure and complete graft coverage (Figs. $1 A 2$ and A3). Due to post-reperfusion edema the fascia could not be closed primarily but was closed four weeks later during a surgical re-exploration for a prolapsed ileostomy. The patient was administered a low-dose dual immunosuppressive regimen of tacrolimus and steroids [19]. No herniation or other skin- or fascia-related complications occurred and at last follow-up -7 years later- she was doing well.

Our second patient was a 34 year-old female (weight: $44 \mathrm{~kg}$ ) with isolated intestinal Churg-Strauss lesions requiring multiple enterectomies resulting in an extreme SBS [20]. Parenteral nutrition resulted in several line infections and ITx was considered the only lifesaving option. As the patient was a non-Eurotransplant resident (Poland) and could not be listed for a cadaveric transplant, the only remaining option was a living-related donation of two meters of distal ileum. The patients' 59 year-old mother (weight: $52 \mathrm{~kg}$; DRWR: 1.2) was a suitable candidate. Since the abdominal domain in the recipient was severely restricted, two TE $\left(400 \mathrm{~cm}^{3} / \mathrm{TE}\right)$ were inserted subcutaneously in each hypochondrium two months prior to the planned ITx. Tissue expansion was uncomplicated and since post-reperfusion edema was limited, primary closure of the skin and fascia could be achieved (Fig. 1B). No abdominal closure-related complications occurred, but due to chronic rejection the graft had to be removed 7 months later.

\subsection{Overall experience}

With inclusion of our 2 patients reported herein, experience with at least $41 \mathrm{TE}$ in 20 ITx candidates has been reported. Median patient age was 2 years 10 months (10 months-56 years) with a median time 
between TE placement and ITx of 6 months ( 2 months-2 years 3 months). Thirteen patients (65\%) finally underwent transplantation (6 cLi-ITX, 6 isolated ITx and 1 multivisceral Tx) of which 12 were from deceased donors and 1 from a living donor. Median DRWR was 1.5 (0.9-3.6). A median of $2(1-3) \mathrm{TE} /$ patient were placed with a median volume/TE of $278 \mathrm{~cm}^{3}\left(118-1000 \mathrm{~cm}^{3}\right)$. In 13 patients (65\%) TE were placed subcutaneously, 1 patient (5\%) was treated with 1 TE placed retromuscularly and 1 TE placed intraperitoneally, in 4 patients (20\%) TE were only inserted intraperitoneally and in 1 case (5\%) a biplanar (intraperitoneally placed and extending retromuscularly) TE was used. In the first case (5\%) reported, TE were placed preperitoneally, without any further details described. All patients required weekly serial percutaneous TE fluid injections, apart from the 2 patients (10\%) reported herein who had osmotic self-inflatable prostheses. Of 18 patients in whom inflatable TE were used, 11 patients (61\%) suffered from TErelated complications of which the most frequent were hematoma and infection due to serial percutaneous injections with even one TErelated death [17]. In the two cases of osmotic expanders, one expander had to be removed due to stoma bag application problems, however no infections or hematoma occurred. Overall TE-related complications occurred in 4 out of 5 patients (80\%) who received an intraperitoneally placed TE and 9 out of 13 patients (69\%) in whom TE were placed subcutaneously. These side-effects resulted in removal of 7 TE from 6 patients before complete expansion was achieved.

In 9 out of 9 patients (100\%) - for whom details on abdominal closure after ITx were reported- primary abdominal closure resulting in graft protection could be obtained. In 2 cases, no details were given on skin or fascia closure. Out of 7 cases, which made a distinction between skin and fascia closure, 4 (57\%) succeeded also in primary fascia closure. In the other 3 cases, alternatives were bridging of the fascia with Alloderm in 1, Gore-Tex in 1 and staged closure in 1 . Two of the latter 3 occurred in patients with subcutaneously placed TE. Although numbers are small, this could be explained by the observation of Watson et al. who revealed - by sequential volumetric computed tomography - that with subcutaneous expansion, the increase in total abdominal volume (delineated by the abdominal skin) was accompanied with a $25 \%$ compensatory intraperitoneal volume loss [16].

\section{Discussion}

Since the first description by Byrd and Hobar in 1989, TE have successfully been used in general, pediatric and plastic surgery for closure of large abdominal fascia and skin defects such as omphalocele, longstanding large abdominal hernias, separation of conjoined twins and reconstructive surgery for extensive burns $[21,22]$. They can be inserted subcutaneously (in front of the anterior rectus fascia), retromuscularly (between the rectus muscle and the posterior fascial sheet) or intraperitoneally (Fig. 2). Despite their versatility, TE have been associated with significant complications since inception. A general complication rate of $13-20 \%$ has been reported with infection, hematoma and device migration being the most frequent $[22,23]$. Experience of TE in patients awaiting ITx is limited due to concerns related to the use of foreign material in critically ill patients as well as space limitations caused by fistulisation, scar tissue and often a stoma.

This review summarizes the experience of 41 TE in 20 ITx candidates. Many of them were children, since TE offer the potential benefit to accept larger grafts and increase the DRWR. An interesting difference in most cases is the TE placement. Three major localisations were identified: intraperitoneally, retromuscular and subcutaneously (Fig. 2).

Although it would seem logical to enlarge the abdominal fascia by intraperitoneally placed TE, this procedure requires more extensive surgery than subcutaneous placement of TE and may potentially result in more severe complications like peritonitis and compression of the intra-abdominal organs. Interestingly, in case of successful intraperitoneal TE placement, of which details of abdominal closure were only available in 2 cases, 1 (50\%) of them did not result in primary fascia closure. Subcutaneous placement in contrast is a relatively benign procedure, does not violate the abdominal cavity, and can be performed with low morbidity. Expansion is generally well tolerated, and large volume increases can be achieved in a relatively short period of time [16]. Moreover, skin is more extensible than fascia and easier to stretch on top of the edematous organs after reperfusion. In the event of a complication, re-exploration of a subcutaneous pocket has significantly less morbidity compared to a laparotomy. The most crucial aspect in the mostly critically ill ITx patient population is to avoid an open abdomen posttransplant and protect the graft, thereby avoiding electrolyte imbalances and limiting the risk for infection. Therefore we would recommend to place TE subcutaneously, providing graft protection at the lowest risk for complications.

To close the fascia, other techniques like component separation or donor fascia transplant can be performed in conjunction with primary skin closure or as a staged procedure. The rationale behind staged closure is to await reperfusion edema to disappear and prevent abdominal compartment syndrome due to tight fascial closure [6,24].

Currently, there is too little evidence on retromuscular placement of TE. Theoretically this would enlarge the abdominal fascia and skin, thereby avoiding intraperitoneal surgery-related complications. However, this procedure, might compromise the potential necessity to perform a component separation technique at a later stage.

Since most TE-related complications were due to serial injections for TE inflation we introduced herein the use of osmotic self-inflatable TE of which the major benefit is to avoid these percutaneous injections. Although our experience is only based on two patients, it seems a safer and more elegant alternative. However, it should be taken into account that in contrast to the volume of inflatable TE -which can be adapted to the tolerance level of the patient- osmotic expansion cannot be controlled. In order to avoid pain and skin tears by overexpansion, an accurate estimation of the maximum volume is crucial. Therefore we advise to opt for relatively small volume osmotic TE $\left(500 \mathrm{~cm}^{3}\right)$ with a maximum of three per patient and adjusted to the location of the stoma and previous incisions.

The limitations of this study are the limited number of cases and the limited number of outcome parameters reported in some studies. Nevertheless, TE might offer significant benefits to ITx candidates in whom primary abdominal closure would be difficult: (i) offering primary abdominal closure and physiological protection of the graft; (ii) avoiding extensive abdominal wall surgery or foreign material insertion peritransplant in a heavily immunosuppressed patient; and (iii) possibility to accept a relatively larger graft, thereby shortening the waiting time for transplantation. Like other abdominal closure techniques, TE bear several potential disadvantages like: (i) prolonged waiting time before transplantation due to placement and expansion; (ii) additional anesthesia exposure; (iii) poor healing of the created pockets in patients under immunosuppression; and (iv) injection-related complications with inflatable TE. Osmotic self-inflatable TE are safer by avoiding serial percutaneous injections.

In the future, we hope that other centers will consider to use the same technique of osmotic self-inflatable TE. We also believe that a multicenter study on the topic of abdominal wall closure would increase our knowledge on the best closure technique for each patient.

\section{Conclusion}

Abdominal tissue expansion is advised for selected ITx candidates with an impaired abdominal wall (skin and fascia) elasticity and limited intra-abdominal domain. Subcutaneous expansion is the most elegant solution and seems the safest option to provide complete skin coverage of the graft, resulting in physiological protection even in the context of large fascial defects. Apart from several benefits, the reported complication rate with conventional inflatable TE is high. Our experience indicates that osmotic self-inflatable TE offer a safer alternative, since they avoid serial percutaneous injections and their associated complications 


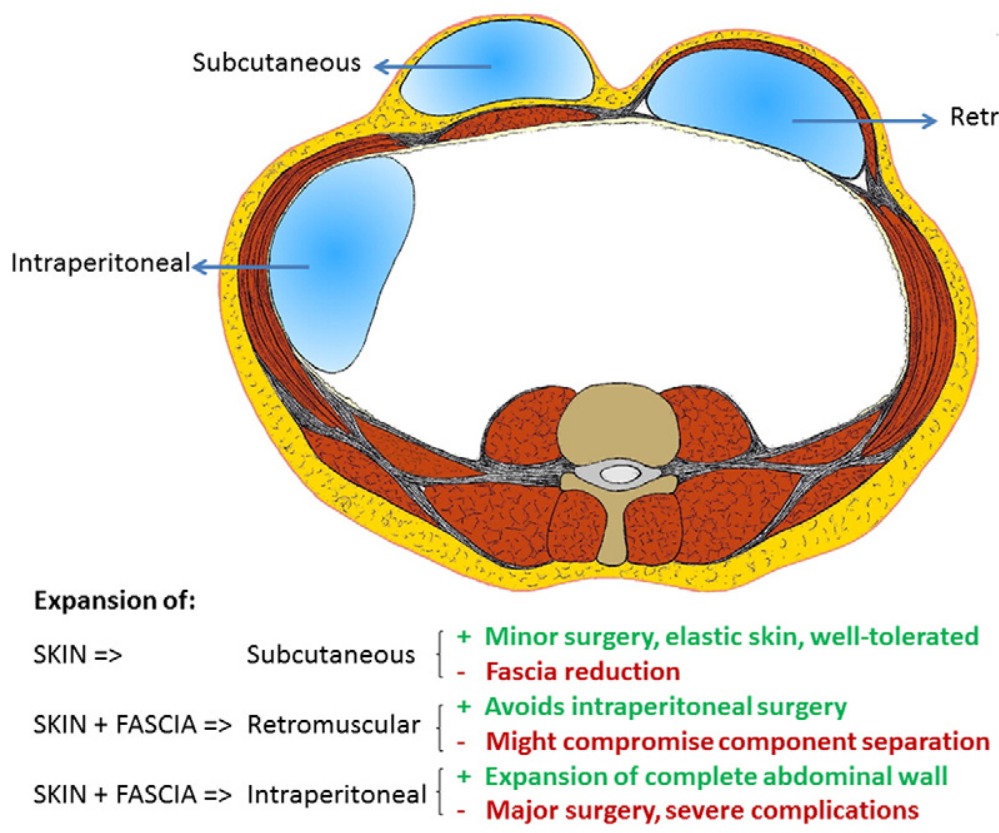

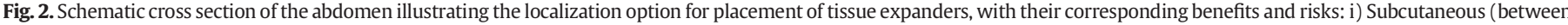
skin and anterior rectus fascia); ii) Retromuscular (between rectus muscle and posterior rectus fascia); and iii) Intraperitoneal. (Illustration by Francesca Maione, MD).

and discomfort, especially in children. Finally, successful expansion may allow the transplantation of larger grafts, thereby reducing the waiting time and mortality on the waiting list of ITx candidates.

\section{Acknowledgement}

The authors of this manuscript have no acknowledgements to report in relation to this manuscript.

Funding source

This research did not receive any specific grant from funding agencies in the public, commercial, or not-for-profit sectors.

Disclosure

The authors of this manuscript have no conflicts of interest to disclose in relation to this manuscript as described by Transplantation Reviews.

We would like to mention following unrelated disclosures: the department of Abdominal Transplant Surgery has received unrestricted grants from Astellas and Roche. Jacques Pirenne and Diethard Monbaliu are holders of a Centrale Afdeling voor Fractionering (CAF) chair in Abdominal Transplant Surgery, Vilvoorde, Belgium. Marc Miserez is consultant for Covidien and has received research grants from Bard, Cook, Covidien and Ethicon.

The authors declare no conflicts of interest.

\section{References}

[1] Abu-Elmagd K. The concept of gut rehabilitation and the future of visceral transplantation. Nat Rev Gastroenterol Hepatol 2015;12:108-20.

[2] Fishbein TM. Intestinal transplantation. N Engl J Med 2009;361:998-1008.

[3] Benedetti E, Panaro F, Holterman M, Abcarian H. Surgical approaches and intestinal transplantation. Best Pract Res Clin Gastroenterol 2003;17:1017-40.

[4] Gerlach UA, Pascher A. Technical advances for abdominal wall closure after intestinal and multivisceral transplantation. Curr Opin Organ Transplant 2012:17:258-67.

[5] Gupte GL, Haghighi KS, Sharif K, et al. Surgical complications after intestinal transplantation in infants and children - UK experience. J Pediatr Surg 2010;45:1473-8.

[6] Sheth J, Sharif K, Lloyd C, et al. Staged abdominal closure after small bowel or multivisceral transplantation. Pediatr Transplant 2012;16:36-40.
[7] International Intestinal Transplantation Registry (ITR). http://www.intestinaltranplant org/itr/. [Accessed May 7th, 2015].

[8] Fecteau A, Atkinson P, Grant D. Early referral is essential for successful pediatric small bowel transplantation: the Canadian experience. J Pediatr Surg 2001;36: 681-4.

[9] Carlsen BT, Farmer DG, Busuttil RW, et al. Incidence and management of abdominal wall defects after intestinal and multivisceral transplantation. Plast Reconstr Surg 2007;119:1247-55

[10] Reyes J, Fishbein T, Bueno J, et al. Reduced-size orthotopic composite liver-intestinal allograft. Transplantation 1998;66:489-92.

[11] Gondolesi G, Selvaggi G, Tzakis A. Use of the abdominal rectus fascia as a nonvascularized allograft for abdominal wall closure after liver, intestinal, and multivisceral transplantation. Transplantation 2009;87:1884-8.

[12] Selvaggi G, Levi DM, Cipriani R, et al. Abdominal wall transplantation: surgical and immunologic aspects. Transplant Proc 2009;41:521-2.

[13] de Ville de Goyet J, Mitchell A, Mayer AD, et al. En bloc combined reduced-liver and small bowel transplants: from large donors to small children. Transplantation 2000; 69:555-9.

[14] Alexandrides IJ, Liu P, Marshall DM, et al. Abdominal wall closure after intestinal transplantation. Plast Reconstr Surg 2000;106:805-12.

[15] Marin-Gutzke M, Mirelis E, Sanchez-Olaso A, et al. Plast Reconstr Surg 2008;121: 359-60.

[16] Watson MJ, Kundu N, Coppa C, et al. Role of tissue expanders in patients with loss of abdominal domain awaiting intestinal transplantation. Transpl Int 2013;26:1184-90.

[17] Vidyadharan R, Van Bommel AC, Kuti K, et al. Use of tissue expansion to facilitate liver and small bowel transplant in young children with contracted abdominal cavities. Pediatr Transplant 2013;17:646-52.

[18] Weiner J, Wu J, Martinez M, et al. The use of bi-planar tissue expanders to augment abdominal domain in a pediatric intestinal transplant recipient. Pediatr Transplant 2014;18:e174-9.

[19] Ceulemans LJ, Braza F, Monbaliu D, et al. The Leuven immunomodulatory protocol promotes T-regulatory cells and substantially prolongs survival after first intestinal transplantation. Am J Transplant 2016. http://dx.doi.org/10.1111/ajt.13815 [Epub ahead of print].

[20] Darius T, Monbaliu D, Aerts R, et al. Living related intestinal transplantation for Churg-Strauss syndrome: a case report. Transplant Proc 2010;42:4423-4.

[21] Byrd HS, Hobar PC. Abdominal wall expansion in congenital defects. Plast Reconstr Surg 1989;84:347-52

[22] Clifton MS, Heiss KF, Keating JJ, et al. Use of tissue expanders in the repair of complex abdominal wall defects. J Pediatr Surg 2011;46:372-7.

[23] LoGiudice J, Gosain AK. Pediatric tissue expansion: indications and complications. ] Craniofac Surg 2003;14:866-72.

[24] Grevious MA, Iqbal R, Raofi V, et al. Staged approach for abdominal closure following combined liver and intestinal transplantation from living donors in pediatric patients. Pediatr Transplant 2009;13:177-81. 